Bernardo L. Horta ${ }^{1}$

\title{
Amamentação e padrões alimentares em crianças de duas coortes de base populacional no Sul do Brasil: tendências e diferenciais
}

\author{
Breastfeeding and feeding patterns in two cohorts \\ of children in southern Brazil: trends and \\ differences
}

Maria Teresa A. Olinto 1

Cesar G. Victora 1

Fernando C. Barros 1

Paula R. V. Guimarães 1

1 Centro de Pesquisas Epidemiológicas, Departamento de Medicina Social, Universidade Federal de Pelotas. C. P. 464, Pelotas, RS, 96030-002, Brasil.

\begin{abstract}
Breastfeeding is fundamental for infant health. However, its median duration in Brazil is very short, and national campaigns since the mid-1980s have attempted to revert this situation. In the present paper, data on breastfeeding are compared for two population-based cohorts of children born in 1982 and 1993 in the city of Pelotas in Southern Brazil. All hospital births in both years were studied and samples of these children were visited at home around 12 months of age. Median duration of breastfeeding increased from 3.1 to 4.0 months in this period. This rise suggests an impact by the national campaigns. In both cohorts, there were interactions between family income and the percentages of children breastfed at different ages. In the early months, breastfeeding was more prevalent in high-income families, but from nine months onwards it was more common among the poor. Low birthweight babies enjoyed shorter duration of breastfeeding. Despite the progress observed during the decade, duration of breastfeeding is still far short of international recommendations, justifying further campaigns prioritizing low birthweight babies and those from low-income families.
\end{abstract}

Key words Breastfeeding; Epidemiology; Diet; Child Health

Resumo A amamentação é fundamental para o crescimento e desenvolvimento da criança. No Brasil, a duração mediana da amamentação é muito baixa; por isso, no início da década de 80, foram implementadas campanhas nacionais de estímulo ao aleitamento materno. O presente artigo teve como objetivo avaliar as mudanças na duração da amamentação na cidade de Pelotas, Rio Grande do Sul, entre os anos de 1982 e 1993. Foram avaliados todos os nascimentos hospitalares ocorridos em ambos os anos, e amostras sistemáticas dessas crianças foram visitadas com cerca de 12 meses de idade. Observou-se um aumento na duração mediana da amamentação de 3,1 meses em 1982 para 4,0 meses em 1993. Este aumento suporta um possível impacto das campanhas nacionais ocorridas durante a década. Em ambas as coortes, houve interação entre a renda familiar e os percentuais de crianças amamentadas em diferentes idades. Nos primeiros meses, estes percentuais foram superiores nas famílias de maior renda, mas, a partir de nove meses, a amamentação foi mais freqüente no grupo de baixa renda. Crianças com baixo peso ao nascer apresentaram durações mais curtas da amamentação. Apesar dos progressos observados, a duração do aleitamento permanece bastante inferior ao recomendado internacionalmente, salientando a necessidade de campanhas futuras que priorizem crianças com baixo peso ao nascer e pertencentes às famílias de baixa renda.

Palavras-chave Amamentação; Epidemiologia; Dieta; Saúde da Criança 


\section{Introdução}

A amamentação desempenha um importante papel no desenvolvimento da criança, principalmente como fator de proteção contra doenças infecciosas. Esta proteção é particularmente importante na diarréia (Victora et al., 1987), que é observada com maior intensidade nos países em desenvolvimento, onde geralmente existe uma maior contaminação ambiental que afeta os alimentos utilizados no desmame das crianças (Jeliffe \& Jeliffe, 1977; Rowland et al., 1978). Além da proteção contra as doenças infecciosas, a amamentação promove o espaçamento intergestacional (Howie, 1991; WHO, 1981) e o crescimento adequado durante os primeiros meses de vida (Dewey et al., 1990).

A duração da amamentação no Brasil é baixa. Pesquisa nacional realizada em 1986 encontrou uma duração mediana de noventa dias (Barros \& Victora, 1990). Apesar de uma série de iniciativas nacionais para o estímulo ao aleitamento, são poucos os estudos com metodologia comparável que permitam uma avaliação das mudanças recentes na duração da amamentação. Um estudo realizado em São Paulo e Recife, baseado em entrevistas realizadas nos serviços de saúde, mostrou aumentos na mediana da amamentação de, respectivamente, 89,4 para 127,5 dias e 65,5 para 104,4 dias, entre os anos de 1981 e 1987 (Rea \& Berquó, 1990; Rea, 1990). No Ceará, dois estudos representativos de base populacional mostraram que, entre 1987 e 1990, a duração mediana do aleitamento aumentou de 124 para 133 dias (Governo do Estado do Ceará \& Unicef, 1992).

Não temos conhecimento sobre outros estudos brasileiros de base populacional que tenham avaliado tendências temporais. O presente estudo tem como objetivo avaliar as mudanças na duração da amamentação na cidade de Pelotas, RS, com base nos estudos longitudinais da população materno-infantil realizados nos anos de 1982 e 1993.

\section{Metodologia}

Como parte do Estudo Longitudinal da População Materno-Infantil de Pelotas, foram avaliados todos os nascimentos hospitalares ocorridos nos anos de 1982 e 1993. A metodologia dos estudos encontra-se descrita em outras publicações (Victora et al., 1989; Barros et al., 1990; Victora et al., 1996). As mães foram entrevistadas logo após o parto e, entre outras variáveis, forneceram informações sobre a renda familiar total, obtida pela soma das rendas indi- viduais no mês anterior à entrevista. Todas as crianças foram pesadas em balanças pediátricas calibradas.

No início de 1983, tentou-se localizar aquelas crianças urbanas nascidas entre janeiro e abril de 1982, que apresentavam então uma idade média de 12 meses. Para a coorte de 1993, uma amostra dos nascimentos foi acompanhada aos seis e 12 meses de idade, incluindo todas as crianças com baixo peso ao nascer $(<2.500 \mathrm{~g})$ e uma amostra sistemática de cerca de $20 \%$ das demais crianças. Para reproduzir a proporção de baixo peso ao nascer na população geral, os resultados finais foram ponderados, com o objetivo de reproduzir na amostra estudada a proporção de baixo peso ao nascer observada no estudo perinatal.

Em ambos os estudos, as mães responderam a um questionário domiciliar aplicado por estudantes universitários das áreas de saúde e serviço social, previamente treinados. Cerca de $5 \%$ das entrevistas foram repetidas por um supervisor para controle da qualidade. O questionário incluiu perguntas sobre a duração do aleitamento materno e as idades em que diferentes tipos de alimentos foram introduzidos regularmente. A idade do desmame foi definida como a interrupção total da amamentação.

Para evitar viés de memória, a comparação sobre a idade do desmame e a introdução de alimentos complementares nas duas coortes baseou-se nos dados colhidos quando as crianças apresentavam cerca de 12 meses de idade. Em 1993, dados sobre a introdução de alimentos no primeiro semestre de vida foram coletados aos seis meses de idade, sendo esta informação utilizada na análise. Quando havia discrepância entre as idades do desmame informados pela mãe aos seis e aos 12 meses, utilizou-se esta última informação para fins de comparabilidade com a coorte de 1982.

O padrão de alimentação das crianças no primeiro ano de vida foi classificado como:

Leite materno predominante: Crianças amamentadas, recebendo ou não outros líquidos, como chás ou água. Não foi possível constituir separadamente uma categoria de crianças amamentadas exclusivamente, isto é, sem qualquer complemento, por ser seu número extremamente reduzido.

Leite materno parcial: Crianças que recebiam leite materno complementado com outros leites, como leite de vaca ou em pó, ou por outros alimentos sólidos ou semi-sólidos.

Desmamadas: Crianças que não recebiam leite materno.

Na análise estatística, utilizou-se o programa SPSS for Windows para calcular o percentual 
de crianças amamentadas em diferentes idades e estimar a duração mediana da amamentação através de tábuas de vida. Na comparação das curvas de sobrevivência da amamentação, fezse uso do teste Log Rank (Kirkwood, 1988).

\section{Resultados}

Em 1983, foram localizados 1.556 (81,8\%) das 1.820 crianças nascidas entre janeiro e abril de 1982, que apresentavam uma idade média de 11,2 meses (desvio padrão 1,3 meses). Em 1993, de uma amostra de 1.460 crianças, foi possível acompanhar 1.414 (96,8\%) e 1.364 $(93,4 \%)$, respectivamente, com seis e 12 meses.

Observou-se um aumento na duração mediana da amamentação, de 3,1 meses para 4,0 meses em 1993 (Tabela 1). A Figura 1 mostra que, embora inicialmente as crianças de 1982 fossem amamentadas em maior proporção, em 1993, já a partir de um mês de idade, as freqüências de amamentação eram superiores.

A Figura 2 mostra o padrão alimentar das crianças menores de um ano, nascidas nas coortes de 1982 e 1993. O lado esquerdo da figura refere-se a 1982 e o direito a 1993. No eixo horizontal, encontram-se as idades em meses (um, três, seis e 12 meses), sendo que, para as

Tabela 1

Mediana da duração da amamentação conforme o gênero, renda familiar e peso de nascimento. Pelotas 1982 e 1993.

\begin{tabular}{|c|c|c|}
\hline & \multicolumn{2}{|c|}{$\begin{array}{c}\text { Mediana da duração } \\
\text { da amamentação (meses) }\end{array}$} \\
\hline & 1982 & 1993 \\
\hline Gênero & NS & $p<0,001$ \\
\hline Masculino & 3,0 & 3,8 \\
\hline Feminino & 3,2 & 4,3 \\
\hline $\begin{array}{l}\text { Renda familiar } \\
\text { em salários mínimos }\end{array}$ & NS & NS \\
\hline 1 & 2,9 & 3,9 \\
\hline $1,1-3$ & 2,9 & 4,1 \\
\hline $3,1-6$ & 3,3 & 3,7 \\
\hline $6,1-10$ & 3,6 & 3,6 \\
\hline$>10$ & 3,4 & 5,3 \\
\hline Peso ao nascer $(\mathrm{g})$ & $p<0,05$ & $p<0,0001$ \\
\hline$<2.000$ & 1,8 & 1,4 \\
\hline $2.000-2.499$ & 2,1 & 3,1 \\
\hline $2.500-2.999$ & 3,0 & 4,0 \\
\hline $3.000-3.499$ & 3,0 & 4,1 \\
\hline 3.500 & 3,5 & 4,5 \\
\hline Total & 3,1 & 4,0 \\
\hline
\end{tabular}

$\mathrm{NS}=\mathrm{p}>0,05$ crianças de 1993, aumentam da direita para a esquerda. O centro da figura é o ponto onde convergem as idades, isto é, tanto as crianças da coorte de 1982 como as de 1993 estão com 12 meses. O eixo vertical expressa o percentual de crianças com cada padrão alimentar (leite materno predominante, leite materno parcial ou desmamada). As áreas no interior do gráfico mostram, em percentuais, o contingente de crianças que, em uma determinada faixa etária, apresentam cada padrão alimentar.

Comparando-se as extremidades laterais da figura, onde as crianças estão com um mês de idade, vê-se que, em 1982, 20\% das crianças já estavam desmamadas, contra $15 \%$ em 1993. A mesma proporção de crianças estavam em aleitamento predominante $(73 \%)$ em ambos os anos. O aleitamento parcial cresceu de $7 \%$ (1982) para $12 \%$ (1993).

Aos três meses, houve claramente uma melhora no padrão de amamentação: cerca de um terço (37\%) das crianças recebiam leite materno predominante em 1982, aumentando para $48 \%$ em 1993. Aos seis meses, cerca de $29 \%$ das crianças recebiam leite materno (predominante ou parcial) em 1982, contra $38 \%$ em 1993, um crescimento de nove pontos percentuais. Esta diferença diminuiu para cinco pontos aos 12 meses (centro da Figura 2).

Figura 1

Percentual de crianças amamentadas em diferentes idades. Pelotas, 1982 e 1993.

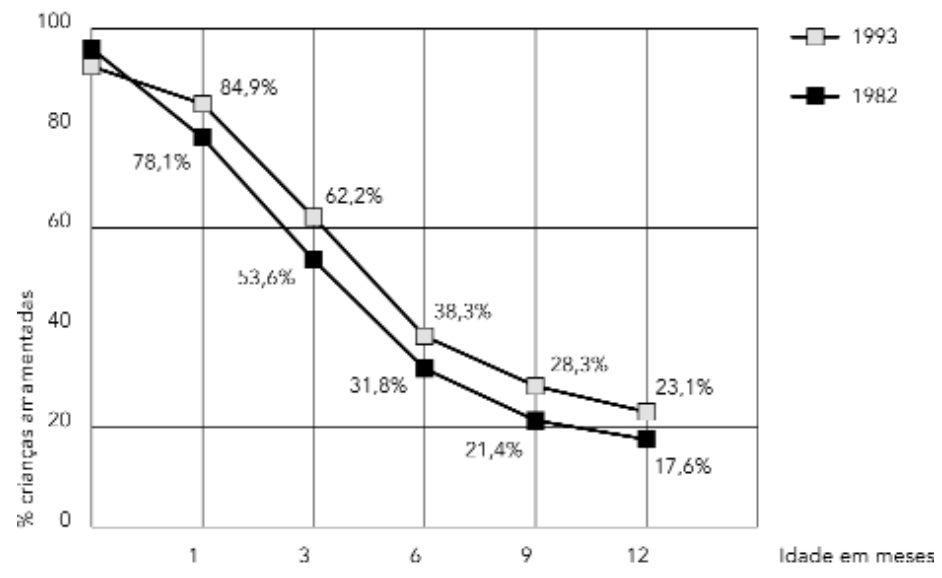


Figura 2

Padrões de amamentação no primeiro ano de vida. Pelotas, 1982 e 1993.

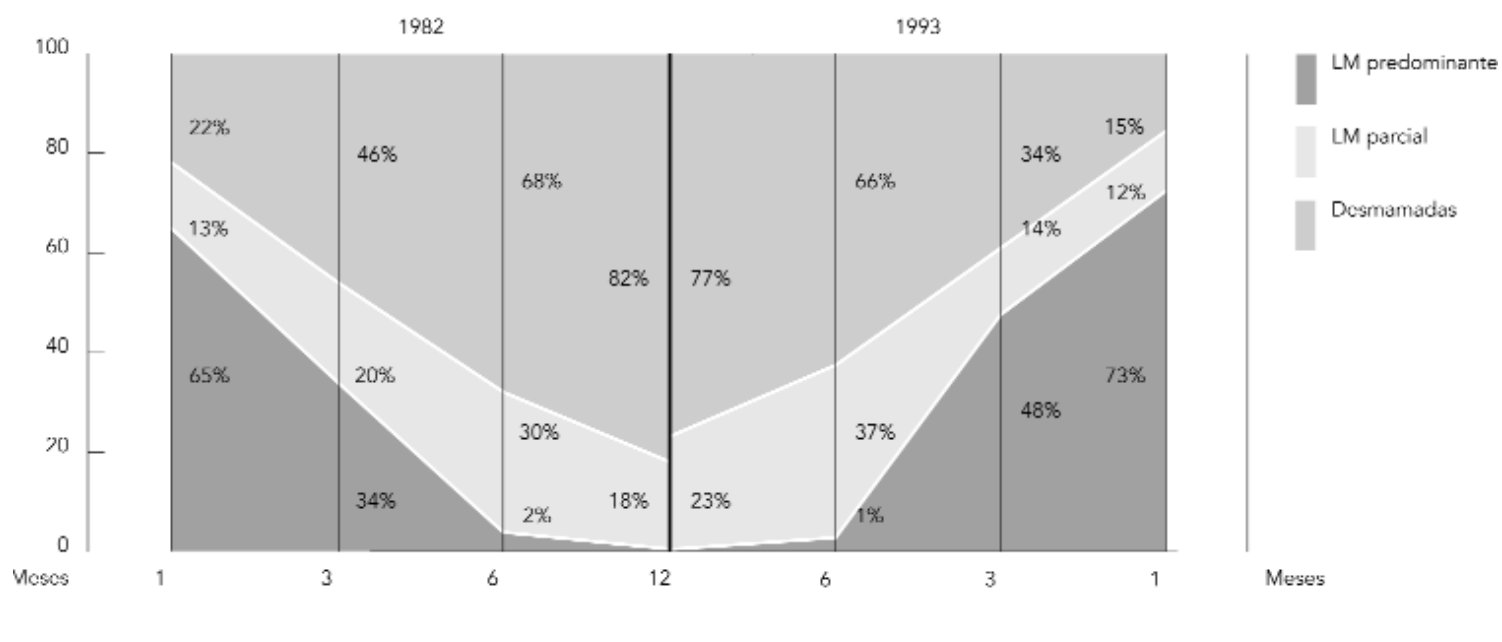

$\mathrm{LM}=$ leite materno

\section{Figura 3}

Percentual de crianças amamentadas em diferentes idades para os grupos extremos de renda. Pelotas, 1982 e 1993.

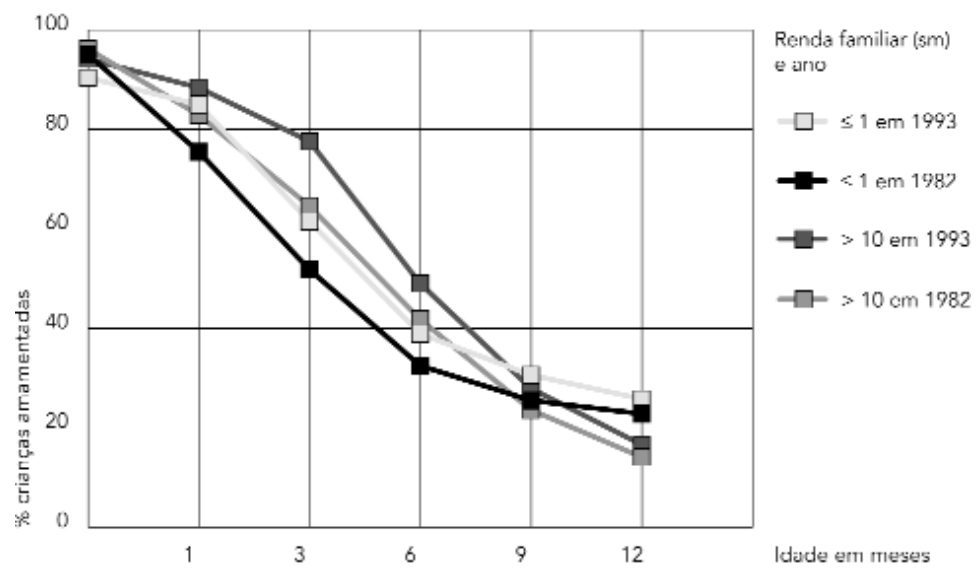

sm = salário mínimo
Durante a década, o aumento na amamentação entre as meninas foi maior do que para os meninos (Tabela 1). Os grupos de mais alta renda familiar apresentaram uma maior duração da amamentação. No tocante ao peso de nascimento, observou-se uma relação diretamente proporcional com a duração da amamentação em ambos os anos, chamando a atenção a curta duração do grupo com menos de $2.000 \mathrm{~g}$ (Tabela 1).

Em ambos os anos, a duração da mediana da amamentação foi maior nos grupos de alta renda do que nos de baixo nível sócio-econômico. Entretanto, quando se analisa o percentual de crianças amamentadas em diferentes idades, observa-se a existência de uma interação com a renda familiar. A Figura 3 mostra os percentuais de crianças amamentadas nos dois grupos extremos de renda conforme a idade, para ambos os anos. Nos primeiros meses, o percentual de crianças amamentadas é maior entre as famílias de alta renda, mas a partir dos nove meses de idade a situação se inverte, sendo mais freqüente a amamentação no grupo de mais baixa renda.

\section{Discussão}

A existência de dois estudos de coorte, com base populacional, permitiu a comparação da evolução dos padrões alimentares em um período de 11 anos. Note-se que os dados refe- 
rentes à coorte de 1982 diferem ligeiramente dos apresentados em publicações anteriores, pois os atuais resultados foram coletados em 1983, quando as crianças nascidas entre janeiro e abril de 1982 tinham cerca de 12 meses em média, enquanto dados publicados dizem respeito a toda a coorte, com a idade média de vinte meses (Victora et al., 1989). Esta diferença é provavelmente devida a viés de memória, pois há evidências de que as mães, com o passar do tempo, tendem a superestimar a duração da amamentação (Huttly et al., 1990).

Observou-se um aumento importante na duração do aleitamento de 3,1 para 4,0 meses. A análise dos padrões alimentares (Figura 2) mostrou ser este aumento devido principalmente ao crescimento do aleitamento predominante (isto é, leite materno mais líquidos) até os três meses de idade, faixa etária em que o efeito protetor é mais marcado (Victora et al., 1987).

A introdução de outros líquidos além do leite materno nesta faixa etária não só é desnecessária - pois crianças amamentadas exclusivamente não necessitam receber água adicional -, como às vezes prejudicial (Victora, 1992). Esse processo pode levar à diminuição da freqüência e intensidade de sucção, reduzindo a produção de leite materno. Há também o risco de infecções pela contaminação de mamadeiras ou dos próprios alimentos. Além disso, alimentação precoce utilizando certos tipos de alimentos, como cereais ou vegetais, podem interferir na absorção de ferro, causando deficiência. Riscos a longo prazo, como obesidade, hipertensão, arteriosclerose e alergia alimentar, também podem estar associados com a introdução de alimentos ao desmame (Jeliffe \& Jeliffe, 1979; Akre, 1989).

A Organização Mundial da Saúde e o Unicef também preconizam que o aleitamento parcial se prolongue até os dois anos de idade, ao passo que, no presente estudo, apenas $23 \%$ das crianças eram amamentadas aos 12 meses. Cabe discutir até que ponto esta última recomendação seria realista para áreas urbanas do Brasil.

Inúmeros fatores atuam na decisão de amamentar, dificultando o estudo do impacto de programas de incentivo à amamentação. Mesmo assim, os presentes dados apóiam um possível impacto do Programa Nacional de Amamentação realizado a partir de $1981 \mathrm{em}$ todo o Brasil (Rea, 1990). Estudos realizados em São Paulo e Recife, embora sem base populacional, confirmam este provável impacto. As práticas hospitalares favoráveis à amamentação mudaram bastante na década, como, por exemplo, alojamento conjunto, contato preco- ce mãe-filho, ausência de mamadeira no berçário. Cabe salientar que, até o presente, não existe na cidade nenhum hospital credenciado pelo programa "Hospital Amigo da Criança". Outra possível explicação seria a grande expansão da rede de postos de atenção básica de saúde no Município (Costa et al., 1996).

Confirmaram-se, ainda, achados anteriores (Victora et al., 1989; Barros et al., 1986 a e b) sobre diferenciais biológicos e sócio-econômicos na amamentação. Crianças de famílias de baixa renda ou nascidas com baixo peso, que mais necessitam do aleitamento, são as que menos recebem leite materno nos primeiros meses de vida. A menor duração da amamentação em crianças de baixo peso ao nascer tem sido encontrada em outros lugares (WHO, 1981). A morbi-mortalidade em recém nascidos de baixo peso é maior naqueles que consomem fórmulas artificiais de leite do que entre os amamentados (Unicef, 1981).

Quanto à renda, os resultados mostraram uma relação paradoxal. Mães de alta renda amamentam mais nos primeiros meses de vida do que as de baixa renda. Esta fase da vida é justamente quando os riscos de morbi-mortalidade são maiores, particularmente para as crianças de famílias pobres, como mostram outros artigos no presente suplemento (Menezes et al., 1996; Cesar et al., 1996). O desmame precoce entre estas crianças pode estar contribuindo para o aumento nos índices de morbimortalidade. Por outro lado, observa-se uma maior prevalência de amamentação entre as crianças pobres aos nove e 12 meses.

É bem conhecida a suscetibilidade do comportamento materno de aleitamento em relação a eventos externos. Nas décadas de 50 a 70, quando houve um grande incentivo ao uso de leite em pó, a duração do aleitamento caiu marcadamente em vários países (Jellife \& Jellife, 1979). Mais recentemente, campanhas de incentivo à amamentação e de valorização do leite materno resultaram em uma reversão nas tendências de queda (Rea, 1990; Jellife \& Jellife, 1979; Barros et al., 1986a). Apesar destas mudanças, o aleitamento materno em nosso meio permanece marcadamente inferior às recomendações internacionais, o que reforça a necessidade de se continuar estimulando a amamentação nos primeiros meses de vida. Os presentes resultados sugerem ainda que campanhas futuras devem ser centradas em crianças de famílias de baixa renda e de baixo peso ao nascer. 


\section{Referências}

AKRE, J., 1989. Infant Feeding: The Physiological Basis. Geneva: World Health Organization.

BARROS, F. C.; VICTORA, C. G. \& VAUGHAN, J. P., 1986a. Breastfeeding and socioeconomic status in Southern Brazil. Acta Paediatrica Scandinavica, 75:558-562.

BARROS, F. C.; VICTORA, C. G.; VAUGHAN, J. P. \& SMITH, P. G., 1986b. Birthweight and duration of breastfeeding are the benefical effects of breastfeeding being overestimated? Pediatrics, 78:656661.

BARROS, F. C. \& VICTORA, C. G., 1990. Breastfeeding Patterns and Determinants in Brazil. New York: Population Council.

BARROS, F. C.; VICTORA, C. G. \& VAUGHAN, J. P., 1990. Strategies for following up 6,000 children in a developing country. Perinatal and Pediatric Epidemiology, 4:267-282.

CESAR, J. A.; VICTORA, C. G.; BARROS, F. C.; RAMOS, F. A.; ALBERNAZ, E. P.; OLIVEIRA, L. O.; HALPERN, R.; BREITENBACH, A.; STONE, M. \& FRACALOSSI, V., 1996. Hospitalizações em menores de um ano pertencentes a duas coortes de base populacional no Sul do Brasil: tendências e diferenciais. Cadernos de Saúde Pública, 12(supl. 1):67-71.

COSTA, J. S. D.; VICTORA, C. G.; BARROS, F. C.; HALPERN, R.; HORTA, B. L. \& MANZOLLI, P., 1996. Assistência médica em duas coortes de base populacional no Sul do Brasil. Cadernos de Saúde Pública, 12(supl. 1):59-66.

DEWEY, K. G.; HEINIG, M. J.; NOMMSEN, L. A. \& LÖNNERDAL, B., 1990. Growth patterns of breastfed infants during the first year of life: The DARLING study. In: Breastfeeding, Nutrition, Infection and Infant Growth in Developed and Emerging Countries (S. A. Atkinson, L. A. Hanson \& R. K. Chandra, eds.), pp. 269-282, St. John's: ARTS.

GOVERNO DO ESTADO DO CEARÁ \& UNICEF, 1992. Crianças e Adolescentes no Ceará: Saúde, Educação e Trabalho. Fortaleza: Governo do Estado do Ceará/UNICEF

HOWIE, P. W., 1991. Breastfeeding: A natural method for childspacing. American Journal of Obstetrics and Gynecology, 165:1900-1901.

HUTTLY, S. R. A.; VICTORA, C. G.; BARROS, F. C.; BERIA, J. U. \& VAUGHAN, J. P., 1990. Do mothers overstimate breastfeeding duration? An example of recall bias from a study in Southern Brazil. American Journal of Epidemiology, 132:572-575.

JELIFFE, D. B. \& JELIFFE, E. F. P., 1977. "Breast is best": modern meanings. New England Journal of Medicine, 297:912-915.
JELIFFE, D. B. \& JELIFFE, E. P., 1979. Human Milk in the Modern World. Oxford: Oxford Medical Publications.

KIRKWOOD, B. R., 1988. Essentials of Medical Statistics. Oxford: Blackwell Scientific Publications.

MENEZES, A. M.; VICTORA, C. G.; BARROS, F. C.; ALBERNAZ, E.; MENEZES, F. S.; JANNKE, H. A.; ALVES, C. \& ROCHA, C., 1996. Mortalidade infantil em duas coortes de base populacional no Sul do Brasil: tendências e diferenciais. Cadernos de Saúde Pública, 12(supl. 1):79-86.

REA, M. F., 1990. The Brazilian national breastfeeding program: a sucess story. International Journal of Gynecology and Obstetrics, 31:79-82.

REA, M. F. \& BERQUÓ, E. S., 1990. Impact of the Brazilian national breast-feeding programme on mothers in greater São Paulo. Bulletin of the World Health Organization, 68:365-371.

ROWLAND, M. G. M.; BARREL, R. A. E. \& WHITEHEAD, R. G., 1978. The weanling's dilemma. Bacterial contamination in traditional Gambian Weaning foods. The Lancet, 1:316-318.

UNICEF, 1981. Breast-feeding and health. Assigment children. Geneva: Unicef.

VICTORA, C. G.; SMITH, P. G.; VAUGHAN, J. P.; NOBRE, L. C.; TEIXEIRA, A. M. B.; FUCHS, S. M. C.; MOREIRA, L. B.; GIGANTE, L. P. \& BARROS, F. C., 1987. Evidence for protection by breast-feeding against infant deaths from infectious diseases in Brazil. The Lancet, 2:319-321.

VICTORA, C. G.; BARROS, F. C. \& VAUGHAN, J. P. 1989. Epidemiologia da Desigualdade: Um Estudo Longitudinal de 6.000 Crianças Brasileiras. São Paulo: Cebes/Hucitec.

VICTORA, C. G., 1992. Exclusive breastfeeding: breast is best... but breast alone is best of all. Dialogue on Diarrhoea, 49:2.

VICTORA, C. G.; BARROS, F. C.; HALPERN, R.; MENEZES, A. M. B.; HORTA, B. L.; TOMASI, E.; WEIDERPASS, E.; CESAR, J. A.; OLINTO, M. T.; GUIMARÃES, P. R. V.; GARCIA, M. M. \& VAUGHAN, J. P., 1996. Estudo longitudinal da população materno-infantil da região urbana do Sul do Brasil, 1993: aspectos metodológicos e resultados preliminares. Revista de Saúde Pública, 30:34-45.

WHO (World Health Organization), 1981. Contemporary patterns of breast-feeding: Report on the WHO Collaborative Study on Breast-feeding. Geneva: WHO. 